\title{
A RELAXATION RESULT FOR AUTONOMOUS INTEGRAL FUNCTIONALS WITH DISCONTINUOUS NON-COERCIVE INTEGRAND
}

\author{
Carlo Mariconda ${ }^{1}$ and Giulia Treu $^{1}$
}

Abstract. Let $L: \mathbb{R}^{N} \times \mathbb{R}^{N} \rightarrow \mathbb{R}$ be a Borelian function and consider the following problems

$$
\begin{gathered}
\inf \left\{F(y)=\int_{a}^{b} L\left(y(t), y^{\prime}(t)\right) \mathrm{d} t: y \in A C\left([a, b], \mathbb{R}^{N}\right), y(a)=A, y(b)=B\right\} \\
\inf \left\{F^{* *}(y)=\int_{a}^{b} L^{* *}\left(y(t), y^{\prime}(t)\right) \mathrm{d} t: y \in A C\left([a, b], \mathbb{R}^{N}\right), y(a)=A, y(b)=B\right\} .
\end{gathered}
$$

We give a sufficient condition, weaker then superlinearity, under which $\inf F=\inf F^{* *}$ if $L$ is just continuous in $x$. We then extend a result of Cellina on the Lipschitz regularity of the minima of $(P)$ when $L$ is not superlinear.

Mathematics Subject Classification. 37N35.

Received June 27, 2003.

\section{INTRODUCTION}

We consider the relationships between the problems

$$
\begin{gathered}
\inf \left\{F(y)=\int_{a}^{b} L\left(y(t), y^{\prime}(t)\right) \mathrm{d} t: y \in A C\left([a, b], \mathbb{R}^{N}\right), y(a)=A, y(b)=B\right\} \\
\inf \left\{F^{* *}(y)=\int_{a}^{b} L^{* *}\left(y(t), y^{\prime}(t)\right) \mathrm{d} t: y \in A C\left([a, b], \mathbb{R}^{N}\right), y(a)=A, y(b)=B\right\} .
\end{gathered}
$$

It is well known that $\inf F=\inf F^{* *}$ if $L$ is super-linear and continuous. Recently Cellina in [5] proved that the same conclusion holds true assuming, instead of superlinearity, a weaker growth condition that we will call $(G A)$. Roughly, a convex function $L(x, \xi)$ satisfies $(G A)$ if the intersection of the supporting hyperplane to its epigraph at $(\xi, L(x, \xi))$ with the ordinate axis tends to $-\infty$ as $|\xi|$ tends to $+\infty$, uniformly with respect to $x$ in compact sets. This condition implies, but is not equivalent to, a sort of conical growth: we say that $L$

Keywords and phrases. Lipschitz, regularity, non-coercive, discontinuous, calculus of variations.

1 Dipartimento di Matematica pura e applicata, Università di Padova, 7 via Belzoni, 35131 Padova, Italy;

e-mail: maricond@math.unipd.it; treu@math.unipd.it

(c) EDP Sciences, SMAI 2004 
satisfies $(C G A)$ if for every $\xi_{0}$ there exist $\varepsilon, R>0$ such that, for every $|\xi| \geq R$,

$$
L(x, \xi) \geq L\left(x, \xi_{0}\right)+p\left(x, \xi_{0}\right) \cdot\left(\xi-\xi_{0}\right)+\varepsilon|\xi|+\text { const. }
$$

whenever $x$ belongs to a prescribed compact set and $p\left(x, \xi_{0}\right)$ belongs to the subdifferential of $\xi \mapsto L(x, \xi)$ in $\xi_{0}$. We weaken here the continuity assumption of $L$ in both variables and we prove that, if $L(x, \xi)$ is just continuous in $x$ and satisfies $(C G A)$, then $\inf F=\inf F^{* *}$.

The proof of the result is based on Theorem 3.2, a uniform approximation of the bipolar of a (discontinuous) function $L(\xi)$ satisfying $(C G A)$ in terms of the convex hull of the graph of $L$; this kind of result is classical when $L$ is supposed to be lower semi-continuous and superlinear in $\xi[7]$.

In the last part of the paper we are concerned with an application to the Lipschitz regularity of the minima of $(P)$. It is well known that, if $L(x, \xi)$ is superlinear and convex in $\xi$, then every minimizer of $(P)$ is Lipschitz. The same result was obtained recently by dropping some of the assumptions: no continuity and no convexity but superlinearity is assumed in [6], continuity, no convexity and assumption $(G A)$ instead of superlinearity is assumed in [5], no continuity and no convexity but the requirement that every section $\lambda \mapsto L(x, \lambda u)(\lambda \geq 0$, $|u|=1)$ satisfies $(G A)$ in [8], extending [6].

As a consequence of our relaxation result we prove that the minima of $(P)$ are Lipschitz if $L(x, \xi)$ is just continuous in $x$ and satisfies $(G A)$, thus extending the main result in [5].

We point out that there are several results concerning the representation of the lower semi-continuous envelope of integral functionals; we just mention $[2,4]$ for some recent results and references. Here we are interested in comparing the values of the infima of problems $(P)$ and $\left(P^{* *}\right)$ instead of establishing a representation formula.

\section{Notation AND PRELIMINARY RESUlts}

In this paper $|\cdot|$ is the Euclidean norm and "." the scalar product in $\mathbb{R}^{N}$. For a function $L(x, \xi): \mathbb{R}^{N} \times \mathbb{R}^{N} \rightarrow \mathbb{R}$ we denote by $L^{* *}(x, \xi)$ (resp. $\partial L^{* *}(x, \xi)$ ) the bipolar (resp. the subdifferential of the bipolar) of $\xi \mapsto L(x, \xi)$. Finally, $A C\left([a, b], \mathbb{R}^{N}\right)$ is the space of absolutely continuous functions on $[a, b]$ with values in $\mathbb{R}^{N}$.

Here $L: \mathbb{R}^{N} \times \mathbb{R}^{N} \longrightarrow \mathbb{R}$ is just a Borelian function. We assume moreover that $L^{* *}(x, \xi) \neq-\infty$ for every $x$ and $\xi$; this is the case, for instance, if $L$ is bounded below by an affine function of $\xi$.

The following growth condition will be assumed in the main result.

Conical growth assumption $(C G A)$. For every compact subset $C$ of $\mathbb{R}^{N}$ and $R_{0} \geq 0$ there exist $\varepsilon>0, R>0$ and $c \in \mathbb{R}$ such that

$$
\forall \xi \in \mathbb{R}^{N} \quad|\xi| \geq R \quad L^{* *}(x, \xi) \geq L^{* *}\left(x, \xi_{0}\right)+p\left(x, \xi_{0}\right) \cdot\left(\xi-\xi_{0}\right)+\varepsilon|\xi|+c
$$

for every $x \in C,\left|\xi_{0}\right| \leq R_{0}$ and $p\left(x, \xi_{0}\right)$ in $\partial L^{* *}\left(x, \xi_{0}\right)$.

The following growth assumption was introduced by Cellina in [5] in the case where $L$ is continuous.

Growth assumption $(G A)$.

We say that $L$ satisfies $(G A)$ if there exist $p(x, \xi)$ in $\partial L^{* *}(x, \xi)$ such that

$$
\lim _{|\xi| \rightarrow+\infty} p(x, \xi) \cdot \xi-L^{* *}(x, \xi)=+\infty
$$

uniformly for $x$ in a compact set.

\section{Remark 2.1.}

i) We point out that, in [5], the definition of $(G A)$ is slightly different: it is formulated in an equivalent way in terms of the polar of $L$ in $(x, p(x, \xi))$; moreover the uniformity with respect to the first variable is not required since it is a consequence of the continuity of $L$. We use it here since we drop the continuity assumption. 
ii) Assumption $(G A)$ is fulfilled if, for instance, $L(x, \xi)$ is superlinear with respect to $\xi$; the proof can be easily done following the lines of [5].

We refer to $[8]$ for a survey on the properties of the functions that satisfy $(G A)$.

Theorem 2.2. [8, Cor 4.4] Assume that $L$ is bounded on compact sets and satisfies the Growth Assumption $(G A)$. Then L satisfies $(C G A)$.

\section{ReLAXATiOn}

It is well known that if $L: \mathbb{R}^{N} \rightarrow \mathbb{R}$ is a function whose bipolar is finite, then, for every $\varepsilon>0$ and $\xi$ in $\mathbb{R}^{N}$, there exists $\xi_{1}, \ldots, \xi_{m}(m \leq N+1)$ in $\mathbb{R}^{N}$ and coefficients of a convex combination $\alpha_{1}, \ldots, \alpha_{m}$ such that $\sum_{i} \alpha_{i} L\left(\xi_{i}\right) \leq L^{* *}(\xi)+\varepsilon$ and $\sum_{i} \alpha_{i} \xi_{i}=\xi$. We prove in the next Theorem 3.2 that if $L$ satisfies $(C G A)$ then, allowing $m \leq 2 N+2$, the points $\xi_{i}$ may be bounded uniformly with respect to $\xi$ in compact sets. For this purpose we first quote, in a more general setting, a powerful consequence of $(C G A)$ that was established in [5] in the continuous case. For every $(x, \xi)$ we set

$$
\bar{L}(x, \xi)=\liminf _{\eta \rightarrow \xi} L(x, \eta),
$$

i.e. $\bar{L}(x, \xi)$ denotes the lower semi-continuous envelope of the map $\eta \mapsto L(x, \eta)$. The proof of the following result is based on the fact that if $f: \mathbb{R}^{N} \rightarrow \mathbb{R}$ is convex and satisfies $(C G A)$ then the intersection of its epigraph with any supporting hyperplane is bounded. This condition is referred in [5] as the Bounded Intersection Property.

Theorem 3.1. Assume that $L$ satisfies $(C G A)$ and let $p(x, \xi) \in \partial L^{* *}(x, \xi)$. Then given $R_{0}>0$ and a compact subset $C$ of $R^{N}$ there exists $R>0$ (depending only on $R_{0}$ and $C$ ) such that for every $x \in C$; for every $\xi$, with $|\xi| \leq R_{0}$, there exist at most $\nu \leq N+1$ points $\xi_{i}$, with $\left|\xi_{i}\right| \leq R$, and coefficients of a convex combination $\alpha_{i}$, such that

$$
\left(\begin{array}{c}
\xi \\
L^{* *}(x, \xi)
\end{array}\right)=\sum_{i=1}^{\nu} \alpha_{i}\left(\begin{array}{c}
\xi_{i} \\
L \\
\left(x, \xi_{i}\right)
\end{array}\right)
$$

and $L^{* *}(x, \xi)=\bar{L}\left(x, \xi_{i}\right)=L^{* *}(x, \xi)+p(x, \xi) \cdot\left(\xi-\xi_{i}\right)$.

Proof. It is enough to remark that Theorem 1 in [5] holds for functions that are lower semi-continuous instead of continuous and that the bipolar of a function coincides with the bipolar of its lower semi-continuous envelope.

We are now ready to state a version of Theorem 3.1 that does not involve the lower semi-continuous envelope of $L$.

Theorem 3.2. Assume that $L(x, \xi)$ satisfies $(C G A)$ and that $L$ is bounded on the compact sets. Then given $R_{0}>0$ and a compact subset $C$ of $R^{N}$, there exists $R>0$ (depending only on $R_{0}$ and $C$ ) such that for every $x$ in $C$, for every $\xi$, with $|\xi| \leq R_{0}$ and $\varepsilon>0$, there exist at most $m \leq 2 N+2$ points $\xi_{i}$, with $\left|\xi_{i}\right| \leq R$, and coefficients of a convex combination $\lambda_{i}$, such that

$$
\left\{\begin{array}{l}
\xi=\sum_{i=1}^{m} \lambda_{i} \xi_{i} \\
\sum_{i=1}^{m} \lambda_{i} L\left(x, \xi_{i}\right) \leq L^{* *}(x, \xi)+\varepsilon .
\end{array}\right.
$$

The proof of the result needs several preliminary steps. For the convenience of the reader we first give a sketch of the proof in the case where $L$ does not depend on $x$.

By Theorem 3.1, for $|\xi| \leq R_{0}$, the point $\left(\xi, L^{* *}(\xi)\right)$ can be written as a convex combination of points $\left(\zeta_{i}, \bar{L}\left(\zeta_{i}\right)\right)$ of the epigraph of the lower semi-continuous envelope of $L(\cdot)$; moreover the $\zeta_{i}$ are uniformly bounded, so that they all lie in a simplex generated by $N+1$ affinely independent points $\eta_{1}, \ldots, \eta_{N+1}$. Now each value $\bar{L}\left(\zeta_{j}\right)$ can be approximated with $L\left(\eta^{j}\right)$ for some $\eta^{j}$ arbitrarily near to $\zeta_{j}$; actually it turns out that for $\varepsilon>0$, if $\left|\eta^{j}-\zeta_{j}\right|$ is sufficiently small, then there is a convex combination of $\left(\eta^{j}, L\left(\eta^{j}\right)\right)$ and $N$ points among the $\left(\eta_{i}, L\left(\eta_{i}\right)\right)$ 's whose 
projection on $\mathbb{R}^{N}$ is $\zeta_{j}$ and whose last coordinate is less than $\bar{L}\left(\zeta_{j}\right)+\varepsilon$. The conclusion follows by writing $\xi$ as a convex combinations of the points $\eta_{i}$ and the $\eta^{j}$ constructed as above.

We first need two technical lemmas. Let, if $S$ is a subset of $\mathbb{R}^{N}$, int $S$ denote its interior and convS its convex hull.

Lemma 3.3. Let $\eta_{1}, \ldots, \eta_{N+1}$ be $N+1$ affinely independent points of $\mathbb{R}^{N}$ and $\eta$ in $\operatorname{int}\left(\operatorname{conv}\left\{\eta_{1}, \ldots, \eta_{N+1}\right\}\right)$, the interior of the simplex whose vertices are $\eta_{1}, \ldots, \eta_{N+1}$. Then:

i) for every $I \subset\{1, \ldots, N+1\}$ of cardinality $|I| \leq N$ the set of points $\left\{\eta, \eta_{i}: i \in I\right\}$ is affinely independent;

ii) for every $\xi \in \operatorname{int}\left(\operatorname{conv}\left\{\eta_{1}, \ldots, \eta_{N+1}\right\}\right)$ there exists a subset $I$ of $\{1, \ldots, N+1\}$ of cardinality $N$ such that $\xi \in \operatorname{conv}\left\{\eta, \eta_{i}: i \in I\right\}$.

Proof of Lemma 3.3. i) It is not restrictive to assume that $I=\{1, \ldots, N\}$. Let

$$
\eta=\sum_{j=1}^{N+1} \lambda_{j} \eta_{j} \quad \lambda_{j}>0 \quad \sum_{j=1}^{N+1} \lambda_{j}=1 .
$$

For every $i \in\{1, \ldots, N\}$ we have

$$
\begin{aligned}
\eta-\eta_{i} & =\sum_{j \neq i} \lambda_{j} \eta_{j}+\left(\lambda_{i}-1\right) \eta_{i} \\
& =\sum_{j \neq i} \lambda_{j}\left(\eta_{j}-\eta_{N+1}\right)+\left(\lambda_{i}-1\right)\left(\eta_{i}-\eta_{N+1}\right)
\end{aligned}
$$

so that, in a matrix notation,

$$
\left[\eta-\eta_{1}, \ldots, \eta-\eta_{N}\right]=\left[\eta_{1}-\eta_{N+1}, \ldots, \eta_{N}-\eta_{N+1}\right](\Lambda-I)
$$

where $I$ is the identity and

$$
\Lambda=\left(\begin{array}{ccc}
\lambda_{1} & \ldots & \lambda_{N} \\
\ldots \ldots & \ldots \\
\lambda_{1} & \ldots & \lambda_{N}
\end{array}\right)
$$

Now $\operatorname{det}(\Lambda-I) \neq 0$ since the eigenvalues of $\Lambda$ are $\lambda_{1}, \ldots, \lambda_{N}$ and $\lambda_{i}<1$ for every $i$, proving i).

Proof of ii). Let

$$
\xi=\alpha_{1} \eta_{1}+\cdots+\alpha_{N+1} \eta_{N+1} \quad \eta=\mu_{1} \eta_{1}+\cdots+\mu_{N+1} \eta_{N+1}
$$

and we may assume that $\alpha_{N+1} / \mu_{N+1}=\min \left\{\alpha_{i} / \mu_{i}: i=1, \ldots, N+1\right\}$ (notice that all the $\mu_{i}$ are strictly positive). Set $c_{N+1}=\alpha_{N+1} / \mu_{N+1}$ and, for $i \in\{1, \ldots, N\}, c_{i}=\alpha_{i}-\mu_{i} c_{N+1}$ : then, for every $i, c_{i} \geq 0$; moreover

$$
\begin{aligned}
\sum_{i=1}^{N+1} c_{i} & =\sum_{i=1}^{N} \alpha_{i}-c_{N+1} \sum_{i=1}^{N} \mu_{i}+c_{N+1} \\
& =1-\alpha_{N+1}-\left(1-\mu_{N+1}\right) c_{N+1}+c_{N+1} \\
& =1-\alpha_{N+1}+\mu_{N+1} c_{N+1}=1
\end{aligned}
$$


and

$$
\begin{aligned}
\sum_{i=1}^{N} c_{i} \eta_{i}+c_{N+1} \eta & =\sum_{i=1}^{N}\left(\alpha_{i}-\mu_{i} c_{N+1}\right) \eta_{i}+c_{N+1} \sum_{i=1}^{N+1} \mu_{i} \eta_{i} \\
& =\sum_{i=1}^{N}\left(\alpha_{i}-\mu_{i} c_{N+1}+\mu_{i} c_{N+1}\right) \eta_{i}+c_{N+1} \mu_{N+1} \eta_{N+1} \\
& =\sum_{i=1}^{N} \alpha_{i} \eta_{i}+\alpha_{N+1} \eta_{N+1}=\xi
\end{aligned}
$$

so that $\xi \in \operatorname{conv}\left\{\eta, \eta_{i}: i \in\{1, \ldots, N\}\right\}$.

Lemma 3.4. Let $\eta_{1}, \ldots, \eta_{N+1}$ be $N+1$ affinely independent points of $\mathbb{R}^{N}$ and let $y_{1}, \ldots, y_{N+1}$ be real numbers and $K>0$. For every $\varepsilon>0$ there exists $\delta>0$ such that for every $\eta, \xi \in \operatorname{int}\left(\operatorname{conv}\left\{\eta_{1}, \ldots, \eta_{N+1}\right\}\right)$ and $y, \beta$ in $[-K, K]$, with $|\eta-\xi|<\delta$ and $|y-\beta|<\delta$, there exists a subset $I$ of $\{1, \ldots, N+1\}$ of cardinality $N$ and coefficients $\lambda, \lambda_{i}(i \in I)$ of a convex combination satisfying

$$
\left\{\begin{array}{l}
\xi=\lambda \eta+\sum_{i \in I} \lambda_{i} \eta_{i} \\
\beta+\varepsilon>\lambda y+\sum_{i \in I} \lambda_{i} y_{i}
\end{array}\right.
$$

Remark 3.5. Geometrically Lemma 3.4 states that given $N+1$ points $\left(\eta_{i}, y_{i}\right)$ of $\mathbb{R}^{N} \times \mathbb{R}$ and $(\xi, \beta)$ in $\mathbb{R}^{N} \times \mathbb{R}$ such that $\xi$ lies in the interior of the convex hull $\Lambda$ of the $\eta_{i}$ s then, given a positive $\varepsilon$, for every point $(\eta, y)$ that is sufficiently near to $(\xi, \beta)$ with $\eta \in \Lambda$ there exist $N$ points among the $\left(\eta_{i}, y_{i}\right)$ s which, together with $(\eta, y)$, generate a $\mathrm{N}$ - dimensional simplex in $\mathbb{R}^{N} \times \mathbb{R}$ whose projection in $\mathbb{R}^{N}$ contains $\xi$ and such that $(\xi, \beta+\varepsilon)$ lies above it.

Proof of Lemma 3.4. For every $I \subset\{1, \ldots, N+1\},|I|=N, y \in \mathbb{R}$ and $\eta \in \Lambda:=\operatorname{int} \operatorname{conv}\left\{\eta_{1}, \ldots, \eta_{N+1}\right\}$ by Lemma 3.3i) there exists a unique hyperplane $z=a^{I}(\eta, y) \cdot \xi+b^{I}(\eta, y)$ containing the points $(\eta, y)$ and $\left(\eta_{i}, y_{i}\right)$ $(i \in I)$. Moreover the coefficients $a^{I}(\eta, y), b^{I}(\eta, y)$ are continuous functions of $(\eta, y)$; in fact from the equations

$$
\left\{\begin{array}{l}
a^{I}(\eta, y) \cdot \eta+b^{I}(\eta, y)=y \\
a^{I}(\eta, y) \cdot \eta_{i}+b^{I}(\eta, y)=y_{i}(i \in I)
\end{array}\right.
$$

we deduce that the vector $a^{I}(\eta, y)$ solves the system

$$
a^{I}(\eta, y) \cdot\left(\eta-\eta_{i}\right)=y-y_{i} \quad(i \in I)
$$

again by Lemma 3.3i) the vectors $\eta-\eta_{i}(i \in I)$ are independent so that the latter system has a unique solution $a^{I}(\eta, y)$ given by Cramer's rule which is a continuous function of $\eta$ and $y$; the continuity of $b^{I}$ follows from the equality $b^{I}(\eta, y)=y-a^{I}(\eta, y) \cdot \eta$.

Set, for every $I \subset\{1, \ldots, N+1\}$,

$$
\varphi^{I}(\eta, y ; \zeta)=a^{I}(\eta, y) \cdot \zeta+b^{I}(\eta, y)
$$

we point out that, by construction, for a fixed $(\eta, y)$ the point $\left(\zeta, \varphi^{I}(\eta, y ; \zeta)\right)$ belongs to the (unique) hyperplane containing the points $(\eta, y)$ and $\left(\eta_{i}, y_{i}\right)(i \in I)$. Since, for every $\zeta \in \Lambda$ and $\beta \in \mathbb{R}$,

$$
\varphi^{I}(\zeta, \beta ; \zeta)=\beta,
$$


then, by the uniform continuity of $\varphi^{I}$ on $\Lambda \times[-K, K] \times \Lambda$, for every $\varepsilon>0$ there exists $\delta>0$ such that, for every subset $I$ of $\{1, \ldots, N+1\}$ of cardinality $N$,

$$
\varphi^{I}(\eta, y ; \zeta)<\beta+\varepsilon \text { whenever } \eta, \zeta \in \Lambda \quad|\eta-\zeta|<\delta \text { and } y, \beta \in[-K, K] \quad|y-\beta|<\delta .
$$

Now fix $\xi$ in $\Lambda$ and $\varepsilon>0$. Let $I \subset\{1, \ldots, N+1\}$ be such that $\xi$ belongs to $\operatorname{conv}\left\{\eta, \eta_{i}: i \in I\right\}$; such a set exists by Lemma 3.3ii). Let $\delta$ be such as in (3.1) and $|\eta-\xi|<\delta,|y-\beta|<\delta$ so that $\varphi^{I}(\eta, y ; \xi)<\beta+\varepsilon$. Then, if we set

$$
\xi=\lambda \eta+\sum_{i \in I} \lambda_{i} \eta_{i}
$$

for some coefficients $\lambda, \lambda_{i}(i \in I)$ of a convex combination, the linearity of $\varphi^{I}$ in the third variable yields

$$
\lambda \varphi^{I}(\eta, y ; \eta)+\sum_{i \in I} \lambda_{i} \varphi^{I}\left(\eta, y ; \eta_{i}\right)<\beta+\varepsilon,
$$

proving the claim since $\varphi^{I}(\eta, y ; \eta)=y$ and $\varphi^{I}\left(\eta, y ; \eta_{i}\right)=y_{i}$.

We are now ready to prove Theorem 3.2.

Proof of Theorem 3.2. Fix $x$ in $C$. By Theorem 3.1 there exists $R_{1}>0$ (depending only on $R_{0}$ and $C$ ), $\zeta_{1}, \ldots, \zeta_{\nu}(\nu \leq N+1)$, with $\left|\zeta_{j}\right| \leq R_{1}$, and coefficients $\alpha_{j}$ of a convex combination satisfying

$$
\left\{\begin{array}{l}
\xi=\sum_{j=1}^{\nu} \alpha_{j} \zeta_{j} \\
L^{* *}(x, \xi)=\sum_{j=1}^{\nu} \alpha_{j} \bar{L}\left(x, \zeta_{j}\right) ;
\end{array}\right.
$$

where $\bar{L}$ denotes as usual the lower semi-continuous envelope of $L(x, \cdot)$. It is not restrictive at this stage to assume that $L(x, \xi)=L(\xi)$. Let $\eta_{1}, \ldots, \eta_{N+1}$ be such that

$$
\left\{\zeta \in \mathbb{R}^{N}:|\zeta| \leq R_{1}\right\} \subset \operatorname{int}\left(\operatorname{conv}\left\{\eta_{1}, \ldots, \eta_{N+1}\right\}\right)
$$

and set

$$
y_{i}=L\left(\eta_{i}\right), \quad i=1, \ldots, N+1, \quad K=\sup \left\{L(\zeta):|\zeta| \leq R_{1}\right\}
$$

Fix $\varepsilon>0$ and $j$ in $\{1, \ldots, \nu\}$; set $\beta=\bar{L}\left(\zeta_{j}\right)$. Correspondingly, let $\delta>0$ satisfy the property stated in Lemma 3.4. By the definition of $\bar{L}$ there exist $\eta^{j} \in \operatorname{int}\left(\operatorname{conv}\left\{\eta_{1}, \ldots, \eta_{N+1}\right\}\right)$ such that

$$
\left|\eta^{j}-\zeta_{j}\right|<\delta \quad \text { and } \quad L\left(\eta^{j}\right) \leq \bar{L}\left(\zeta_{j}\right)+\delta .
$$

We apply Lemma 3.4 with $\eta=\eta^{j}, \xi=\zeta_{j}$ and $y=L\left(\eta^{j}\right)$ : there exists a subset $I_{j}$ of $\{1, \ldots, N+1\}$ of cardinality $N$ and coefficients $\lambda^{j}, \lambda_{i}^{j},\left(i \in I_{j}\right)$, such that

$$
\left\{\begin{array}{l}
\zeta_{j}=\lambda^{j} \eta^{j}+\sum_{i \in I_{j}} \lambda_{i}^{j} \eta_{i} \\
\lambda^{j} L\left(\eta^{j}\right)+\sum_{i \in I_{j}} \lambda_{i}^{j} L\left(\eta_{i}\right) \leq \bar{L}\left(\zeta_{j}\right)+\varepsilon .
\end{array}\right.
$$

Therefore we obtain that

$$
\begin{aligned}
\xi=\sum_{j=1}^{\nu} \alpha_{j} \zeta_{j} & =\sum_{j=1}^{\nu} \alpha_{j}\left(\lambda^{j} \eta^{j}+\sum_{i \in I_{j}} \lambda_{i}^{j} \eta_{i}\right) \\
& =\sum_{j=1}^{\nu} \alpha_{j} \lambda^{j} \eta^{j}+\sum_{i \in I_{j}}\left(\sum_{j=1}^{\nu} \alpha_{j} \lambda_{i}^{j}\right) \eta_{i}
\end{aligned}
$$


and moreover

$$
\begin{aligned}
L^{* *}(\xi)+\varepsilon & =\sum_{j=1}^{\nu} \alpha_{j}\left(\bar{L}\left(\zeta_{j}\right)+\varepsilon\right) \\
& \geq \sum_{j=1}^{\nu} \alpha_{j}\left(\lambda^{j} L\left(\eta^{j}\right)+\sum_{i \in I_{j}} \lambda_{i}^{j} L\left(\eta_{i}\right)\right) \\
& =\sum_{j=1}^{\nu} \alpha_{j} \lambda^{j} L\left(\eta^{j}\right)+\sum_{i \in I_{j}}\left(\sum_{j=1}^{\nu} \alpha_{j} \lambda_{i}^{j}\right) L\left(\eta_{i}\right) .
\end{aligned}
$$

If we set

$$
\begin{cases}\lambda_{i}=\alpha_{i} \lambda^{i} & \text { if } i \in\{1, \ldots, \nu\} \\ \lambda_{i}=\sum_{j} \alpha_{j} \lambda_{i-\nu}^{j} & \text { if } i \in\{\nu+1, \ldots, \nu+(N+1)\}\end{cases}
$$

the above formulae can be rewritten as

$$
\left\{\begin{array}{l}
\xi=\sum_{i \leq \nu} \lambda_{i} \eta^{i}+\sum_{i>\nu} \lambda_{i} \eta_{i} \\
\sum_{i \leq \nu} \lambda_{i} L\left(\eta^{i}\right)+\sum_{i>\nu} \lambda_{i} L\left(\eta_{i}\right) \geq L^{* *}(\xi)+\varepsilon .
\end{array}\right.
$$

Moreover $\left|\eta^{i}\right| \leq R$ and $\left|\eta_{i}\right| \leq R$, where $R=\max \left\{\left|\eta_{i}\right|: i=1, \ldots, N+1\right\}$ (which depends only on $R_{1}$ and therefore only on $R_{0}$ and $C$ ); proving the claim.

We consider here the problems

$$
\begin{gathered}
\inf \left\{F(y)=\int_{a}^{b} L\left(y(t), y^{\prime}(t)\right) \mathrm{d} t: y \in A C\left([a, b], \mathbb{R}^{N}\right), y(a)=A, y(b)=B\right\} \\
\inf \left\{F^{* *}(y)=\int_{a}^{b} L^{* *}\left(y(t), y^{\prime}(t)\right) \mathrm{d} t: y \in A C\left([a, b], \mathbb{R}^{N}\right), y(a)=A, y(b)=B\right\} .
\end{gathered}
$$

It is well known that $\inf F=\inf F^{* *}$ if $L$ is continuous and superlinear ([7], Th. IX.3.1); actually in this case $F^{* *}$ is the relaxed functional of $F$. In [5] Cellina proved that $\inf F=\inf F^{* *}$ if $L$ is just continuous and satisfies $(G A)$. We examine here the case where $L$ is just continuous in the first variable, focusing our attention on the infima of the functionals $F$ and $F^{* *}$ instead on the relaxed functional of $F$.

Theorem 3.6. Assume that $L$ is bounded on compact sets and that $x \mapsto L(x, \xi)$ is continuous for every $\xi \in \mathbb{R}^{N}$. If $L$ satisfies $(C G A)$ then $\inf F=\inf F^{* *}$.

Proof. We follow the lines of the proof of the analogous result ([5], Th. 3) in the case where $L$ is continuous in both variables, but instead of Theorem 3.1 we use Theorem 3.2. Let $x \in A C\left([a, b], \mathbb{R}^{N}\right)$ and $\varepsilon>0$. From Theorem 2.4 and Remark 2.8 of [1] applied to $L^{* *}$ there exists a Lipschitz function $x_{R_{0}}$ of Lipschitz constant $R_{0}$ satisfying the boundary conditions and such that

$$
\int_{a}^{b} L^{* *}\left(x_{R_{0}}(t), x_{R_{0}}^{\prime}(t)\right) \mathrm{d} t \leq \int_{a}^{b} L^{* *}\left(x(t), x^{\prime}(t)\right) \mathrm{d} t+\varepsilon / 3 .
$$

Set $C=\left\{x_{R_{0}}(t): t \in[a, b]\right\}$. Since $\left|x_{R_{0}}^{\prime}(t)\right| \leq R_{0}$ for a.e. $t$ then, by Theorem 3.2, there exists $R$ (depending only on $R_{0}$ and $\left.C\right), m \leq 2 N+2$ coefficients $\lambda_{i}(t)$ of a convex combination and vectors $y_{i}(t)(i=1, \ldots, m)$ with 
$\left|y_{i}(t)\right| \leq \mathbb{R}$ such that

$$
\left\{\begin{array}{l}
x_{R_{0}}^{\prime}(t)=\sum_{i=1}^{m} \lambda_{i}(t) y_{i}(t) \\
\sum_{i=1}^{m} \lambda_{i}(t) L\left(x_{R_{0}}(t), y_{i}(t)\right) \leq L^{* *}\left(x_{R_{0}}(t), x_{R_{0}}^{\prime}(t)\right)+\frac{\varepsilon}{3(b-a)}
\end{array}\right.
$$

By a standard selection argument, we may assume that the maps $y_{i}$ and $\lambda_{i}$ are measurable. Fix an integer $k$ and consider the intervals $I_{j}=\left[t_{j}, t_{j+1}\right]$, where $t_{j}=a+j \frac{b-a}{k}(j=0, \ldots, k-1)$ and call $\chi_{I_{j}}$ their characteristic function. By Lyapunov's Theorem on the range of vector measures [9] there exists a partition of $[a, b]$ into $m$ measurable subsets $E_{i}$, with characteristic functions $\chi_{E_{i}}$, such that, for $j=0, \ldots, k-1$, one has

$$
\begin{aligned}
\int_{I_{j}} \sum_{i=1}^{m} \lambda_{i}(t) y_{i}(t) \mathrm{d} t & =\int_{I_{j}} \sum_{i=1}^{m} \chi_{E_{i}}(t) y_{i}(t) \mathrm{d} t \\
\int_{I_{j}} \sum_{i=1}^{m} \lambda_{i}(t) L\left(x_{R_{0}}(t), y_{i}(t)\right) \mathrm{d} t & =\int_{I_{j}} \sum_{i=1}^{m} \chi_{E_{i}}(t) L\left(x_{R_{0}}(t), y_{i}(t)\right) \mathrm{d} t .
\end{aligned}
$$

Denote by $x_{k}$ the absolutely continuous defined by $x_{k}(a)=A$ and

$$
x_{k}^{\prime}(t)=\int_{a}^{t} \sum_{i, j} y_{i}(s) \chi_{I_{j} \cap E_{i}}(s) \mathrm{d} s
$$

in particular for every $k$ and every $j=1, \ldots, k$, we have

$$
\int_{I_{j}} x_{R_{0}}^{\prime}(t) \mathrm{d} t=\int_{I_{j}} x_{k}^{\prime}(t) \mathrm{d} t
$$

so that the functions $x_{R_{0}}$ and $x_{k}$ coincide at each point $t_{j}$. Since

$$
L\left(x_{R_{0}}(t), x_{k}^{\prime}(t)\right)=\sum_{i, j} \chi_{I_{j} \cap E_{i}}(t) L\left(x_{R_{0}}(t), y_{i}(t)\right)
$$

we also have that

$$
\int_{a}^{b} L\left(x_{R_{0}}(t), x_{k}^{\prime}(t)\right) \mathrm{d} t=\int_{a}^{b} \sum_{i=1}^{m} \lambda_{i}(t) L\left(x_{R_{0}}(t), y_{i}(t)\right) \mathrm{d} t
$$

so that, from $\approx$, it follows that

$$
\int_{a}^{b} L\left(x_{R_{0}}(t), x_{k}^{\prime}(t)\right) \mathrm{d} t \leq \int_{a}^{b} L^{* *}\left(x_{R_{0}}(t), x_{R_{0}}^{\prime}(t)\right) \mathrm{d} t+\varepsilon / 3
$$

Now

$$
\int_{a}^{b} L\left(x_{R_{0}}(t), x_{k}^{\prime}(t)\right) \mathrm{d} t=\int_{a}^{b} L\left(x_{k}(t), x_{k}^{\prime}(t)\right) \mathrm{d} t+\int_{a}^{b} L\left(x_{R_{0}}(t), x_{k}^{\prime}(t)\right)-L\left(x_{k}(t), x_{k}^{\prime}(t)\right) \mathrm{d} t
$$

moreover, $x_{R_{0}}$ is uniformly continuous, the functions $x_{k}$ are equi-Lipschitz, $x_{k}\left(t_{j}\right)=x_{R_{0}}\left(t_{j}\right)(j=0, \ldots, k-1)$. Hence, if $t \in[a, b]$ and $t_{j} \leq t \leq t_{j+1}$,

$$
\begin{aligned}
\left|x_{k}(t)-x_{R_{0}}(t)\right| & \leq\left|x_{k}(t)-x_{k}\left(t_{j}\right)\right|+\left|x_{k}\left(t_{j}\right)-x_{R_{0}}\left(t_{j}\right)\right|+\left|x_{R_{0}}\left(t_{j}\right)-x_{R_{0}}(t)\right| \\
& =\left|x_{k}(t)-x_{k}\left(t_{j}\right)\right|+\left|x_{R_{0}}\left(t_{j}\right)-x_{R_{0}}(t)\right| \leq\left(R+R_{0}\right)(b-a) / k
\end{aligned}
$$


so that $x_{k}$ converges uniformly to $x_{R_{0}}$ as $k$ tends to $+\infty$. By our assumption the function $L\left(x_{R_{0}}, x_{k}^{\prime}\right)-L\left(x_{k}, x_{k}^{\prime}\right)$ is bounded a.e. by a constant that does not depend on $k$. The continuity of $L$ with respect to the first variable together with the dominated convergence theorem imply that

$$
\lim _{k \rightarrow+\infty} \int_{a}^{b} L\left(x_{R_{0}}(t), x_{k}^{\prime}(t)\right)-L\left(x_{k}(t), x_{k}^{\prime}(t)\right) \mathrm{d} t=0 .
$$

It follows that for $k$ sufficiently large,

$$
\int L\left(x_{k}(t), x_{k}^{\prime}(t)\right) \mathrm{d} t \leq \int_{a}^{b} L\left(x_{R_{0}}(t), x_{k}^{\prime}(t)\right) \mathrm{d} t+\varepsilon / 3 \leq \int_{a}^{b} L\left(x(t), x^{\prime}(t)\right) \mathrm{d} t+\varepsilon
$$

proving that $\inf F \leq \inf F^{* *}$.

We point out that, under the assumptions of Theorem 3.6, the functional $F^{* *}$ is not in general the relaxed functional of $F$; we refer to [2] for some recent results in this direction. This is the case in the forthcoming example, where we also show that the conclusion of Theorem 3.6 does not hold if $L$ is not continuous in $x$.

Example 3.7. Let $g$ be the characteristic function of $\mathbb{R} \backslash\{0\}$ and $h(\xi)=\xi^{2}$ if $\xi \neq 0, h(0)=1$ and set $L(x, \xi)=g(x)+h(\xi)$. Let $(P),\left(P^{* *}\right)$ be the problems

$$
\begin{aligned}
& \inf \left\{F(y)=\int_{0}^{1} L\left(y(t), y^{\prime}(t)\right) \mathrm{d} t ; \quad y(0)=0, y(1)=0, y \in A C([0,1], \mathbb{R})\right\} \\
& \inf \left\{F^{* *}(y)=\int_{0}^{1} L^{* *}\left(y(t), y^{\prime}(t)\right) \mathrm{d} t ; \quad y(0)=0, y(1)=0, y \in A C([0,1], \mathbb{R})\right\} .
\end{aligned}
$$

For every $x$ in $\mathbb{R}$ we have $L^{* *}(x, \xi)=g(x)+\xi^{2}$, so that the minimum of the problem $\left(P^{* *}\right)$ is equal to 0 and it is obviously assumed for $y(t)=0$. However inf $F \geq 1$; in fact let $y \in A C([0,1], \mathbb{R})$ and set $E=\{t \in[0,1]$ : $y(t)=0\}$, then $y^{\prime}(t)=0$ a.e. on $E$, so that

$$
\begin{aligned}
\int_{0}^{1} L\left(y(t), y^{\prime}(t)\right) \mathrm{d} t & =\int_{E} L(0,0) \mathrm{d} t+\int_{[0,1] \backslash E} L\left(y(t), y^{\prime}(t)\right) \mathrm{d} t \\
& \geq \int_{E} 1 \mathrm{~d} t+\int_{[0,1] \backslash E} g(y(t)) \mathrm{d} t \\
& \geq|E|+|[0,1] \backslash E|=1 .
\end{aligned}
$$

Notice that nevertheless, from [3], the minima of $F$ are Lipschitz.

\section{Lipschitz REgularity of the minima of $(P)$}

In this section we apply our result to the problem of the Lipschitz regularity of the minima of $(P)$. It is well known that if $L(x, \xi)$ is continuous, convex and superlinear in $\xi$ then every minimum of $(P)$ is Lipschitz. In some recent papers the same conclusion is proved under weaker assumptions; we just mention $[5,6,8]$. Our result is in the same spirit of the last two that we recall here.

Theorem 4.1. [5] Assume that $L(x, \xi)$ is continuous in both variables and satisfies $(G A)$. Then every minimizer of $(P)$ in $A C\left([a, b], \mathbb{R}^{N}\right)$ is Lipschitz.

Theorem 4.2. [8] Assume that $L(x, \xi)$ is convex in $\xi$ and satisfies $(G A)$. Then every minimizer of $(P)$ in $A C\left([a, b], \mathbb{R}^{N}\right)$ is Lipschitz.

The following theorem weakens the continuity assumption of Theorem 4.1. 
Theorem 4.3. Assume that $x \mapsto L(x, \xi)$ is continuous for every $\xi$ and that $L$ satisfies $(G A)$. Then every minimizer of $(P)$ in $A C\left([a, b], \mathbb{R}^{N}\right)$ is Lipschitz.

Proof. By Theorem 3.6, $\inf F=\inf F^{* *}$; therefore every minimum of $F$ is a minimum of $F^{* *}$. The function $L^{* *}(x, \xi)$ is convex in $\xi$ and satisfies $(G A)$ : Theorem 4.2 yields the conclusion.

\section{REFERENCES}

[1] G. Alberti and F. Serra Cassano, Non-occurrence of gap for one-dimensional autonomous functionals. Ser. Adv. Math. Appl. Sci. Calculus of variations, homogenization and continuum mechanics 18 (1993) 1-17.

[2] M. Amar, G. Bellettini and S. Venturini, Integral representation of functionals defined on curves of $W^{1, p}$. Proc. R. Soc. Edinb. Sect. A 128 (1998) 193-217.

[3] L. Ambrosio, O. Ascenzi and G. Buttazzo, Lipschitz regularity for minimizers of integral functionals with highly discontinuous integrands. J. Math. Anal. Appl. 142 (1989) 301-316.

[4] G. Buttazzo, Semicontinuity, relaxation and integral representation in the calculus of variations. Pitman Res. Notes Math. Ser. 207 (1989).

[5] A. Cellina, The classical problem of the calculus of variations in the autonomous case: Relaxation and lipschitzianity of solutions. Preprint (2001).

[6] G. Dal Maso and H. Frankowska, Autonomous Integral Functionals with Discontinuous Nonconvex Integrands: Lipschitz Regularity of Minimizers, DuBois-Reymond Necessary Conditions, and Hamilton-Jacobi Equations. Preprint (2002).

[7] I. Ekeland and R. Témam, Convex analysis and variational problems. Classics Appl. Math. 28 (1999).

[8] C. Mariconda and G. Treu, Lipschitz regularity of the minimizers of autonomous integral functionals with discontinuous nonconvex integrands of slow growth. Dipartimento di Matematica pura e applicata, Università di Padova 10 (2003) preprint.

[9] W. Rudin, Functional analysis. International Series in Pure and Applied Mathematics, McGraw-Hill, Inc., New York (1991). 